\section{Three unusual cases of late pacemaker lead malfunction in dogs -2 electrical and 1 mechanical}

\section{Xavier Navarro-Cubas1, João Neves1, Brigite Pedro1, Joanna Dukes-McEwan1, Anna Gelzer2, Marc S. Kraus2}

1 University of Liverpool, Liverpool, UK

2 University of Pennsylvania, Philadelphia, USA

Cardiac pacemaker implantation is the standard treatment for symptomatic bradyarrhythmias.

Common early complications following pacemaker implantation described in dogs include lead dislodgement or migration, lead perforation, infection, hematoma or seroma formation at the pulse generator (PG) pocket, or $\mathrm{PG}$ migration. In addition, mechanical or electrical malfunction of the PG and/or electrode can also occur. Complications of failure to capture and/or sensing can often be dealt with reprogramming.

We present 3 unusual, late complications associated with pacemaker system malfunction.

Dog 1 presented for sudden collapse, 3 years after initial pacemaker implantation (VVIR bipolar, active fixation) for atrial standstill. The ECG demonstrated long periods of asystole, without pacing spikes. Pacemaker interrogation revealed "make-break" signals (MBS) in the intracardiac electrogram, not previously reported in veterinary medicine. These are intermittent, non-physiologic low amplitude, high-frequency signals, not detectable on surface ECG but over-sensed by the pacemaker, resulting in inhibition of the pacing activity. In addition, intermittently increased impedance (between 630 and 2238 $\Omega$ ) was observed with external manipulation of the PG site. Causes of MBS include loose set-screw, partial conductor fracture, failure of internal insulation of bipolar lead, loose active fixation screw, and interaction between active and abandoned leads. Sensitivity reprogramming was not effective in preventing oversensing of MBS. Reprogramming from bipolar to unipolar mode achieved a decrease in the frequency of MBS, but electrode replacement or correction of the loose connection is considered the definitive treatment.

Dog 2 was re-evaluated for collapse episodes occurring one-year post-pacemaker implantation (VVIR bipolar, active fixation) for atrial standstill. Pacemaker interrogation revealed loss of capture. Capture could not be achieved with increasing the pacing amplitude. Reprogramming the pacemaker settings to unipolar resulted in adequate capture. We surmise the loss of capture was due to electrode malfunction, specifically the positive pole (anode) of the pacing circuit.

Dog 3 presented for syncope 2 years after epicardial lead placement (VVIR). Radiographs indicated lead fracture at the interface of the "fish-hook" electrode, confirmed at surgery for lead replacement. Lead conductor fracture is the most common cause of an open circuit, associated with infinitely high lead impedance, as was documented in this case $(>3,000 \Omega)$.

In conclusion, unusual, late pacemaker complications may be diagnosed by thorough pacemaker interrogation. Pacemaker reprogramming may correct or alleviate some of the electrode malfunctions, but definitive treatment of electrode fracture and MBS is lead replacement. 